\title{
Nutritional assessment and length of hospital stay
}

\author{
Ursula G. Kyle MS RD/LD, Jorge A. Coss-Bu MD
}

Previously published at www.cmaj.ca

$\infty \infty$

See related research article by Caccialanza and colleagues, page 1843

$\mathrm{W}$ e have intuitively known for a long time that poor nutritional status in disease is associated with morbidity and mortality. Only recently, however, have studies provided evidence of an association between poor nutritional status and prolonged hospital stay, decreased quality of life, and increased morbidity and mortality. ${ }^{1-3}$ In this issue, the study by Caccialanza and colleagues adds a piece to the puzzle. ${ }^{4}$ The authors assessed the nutritional status, using the Nutritional Risk Index (NRI), ${ }^{5}$ of 1274 ambulatory adult patients at the time of hospital admission and collected clinical information during their hospital stay until death or discharge. They found that nutritional risk at admission and worsening nutritional status during the hospital stay were associated with a prolonged hospital stay.

A number of other studies have confirmed the association between length of stay and nutritional status, ${ }^{1,2}$ and between nutritional risk and complications and mortality. ${ }^{3}$ Although a previous study showed that the Nutritional Risk Index has a lower sensitivity and specificity than the Nutritional Risk Screening Tool 2002 (NRS-2002), ${ }^{6,7}$ a significant association between length of stay and nutritional status has been shown with both of these instruments, ${ }^{5,7}$ and with the Subjective Global Assessment (SGA) ${ }^{8}$ and the Malnutrition Universal Screening Tool (MUST). ${ }^{9}$

The reported prevalence of poor nutritional status has varied widely in studies, in part because of different populations and types of institutions studied, and because of different diagnostic criteria or tools used to define nutritional status. ${ }^{10}$ We need to distinguish between nutritional screening and nutritional assessment.

The purpose of nutritional screening is to rapidly identify patients who are at high nutritional risk or have poor nutritional status at hospital admission. Information is collected on the patient's change in weight, body mass index or weight history, the adequacy of food intake and, with some tools, the severity of disease. Examples of nutritional screening tools are the Malnutrition Universal Screening Tool, the Nutritional Risk Screening Tool 2002, the Nutritional Risk Index and the Mini Nutritional Assessment full and short forms (MNA and MNA$\mathrm{SF}) .{ }^{11}$ These screening tools can be administered by nonprofessional hospital staff, who then refer patients at risk to the registered dietitian or other professional staff for intervention.

The purpose of nutritional assessment, on the other hand, is to define a patient's nutritional status, to identify clinically relevant malnutrition and to monitor changes in the patient's nutritional status. It records anthropometric, dietary and bio-

\section{Key points}

- The purpose of nutritional screening is to rapidly identify patients at high nutritional risk.

- The purpose of nutritional assessment, however, is to define a patient's nutritional status, to define clinically relevant malnutrition and to monitor changes in nutritional status.

- Nutritional risk continues to be unrecognized and undertreated in clinical practice.

- Hospitals need to develop comprehensive strategies for identifying and treating malnutrition at hospital admission and for monitoring the nutritional status of patients during their hospital stay.

chemical measurements, clinical history, findings at physical examination and other parameters. The Subjective Global Assessment and the Mini Nutritional Assessment are nutritional assessment tools. An advantage of nutritional screening tools over nutritional assessment tools is that they require less training to administer them. ${ }^{6}$ The choice of tool depends on the type of hospital, the population to be screened or assessed and the resources available.

One of the problems with both nutritional screening and assessment is that disease and nutritional status interact. Conditions associated with chronic disease, such as anorexia and asthenia, will result in poor food intake, and ultimately protein-energy malnutrition. The malnutrition may increase gastrointestinal dysfunction, cause infection and impair wound healing, which can lead to worse food intake and malnutrition. Many acute events, such as sepsis and pneumonia, may lead to hypermetabolism and severe inflammatory response, and ultimately stress-induced catabolism. Stress-induced catabolism may also cause gastrointestinal dysfunction, infection and impaired wound healing, which can lead to decreased food intake and protein-energy malnutrition. Thus, both chronic and acute conditions can synergistically interact with a patient's nutritional status and lead to progressive malnutrition and prolonged hospital stay. ${ }^{12}$

The recent literature suggests that simple anthropometric parameters (weight and body mass index) underestimate the nutritional risk of patients admitted to hospital. ${ }^{12}$ Recent weight loss appears to be the most important single indicator of nutri-

Ursula Kyle and Jorge Coss-Bu are with the Department of Pediatrics, Critical Care Medicine, Baylor College of Medicine, Houston, USA.

CMAJ 2010. DOI:10.1503/cmaj.101256 
tional status. ${ }^{12}$ The Malnutrition Universal Screening Tool and the Nutritional Risk Screening Tool 2002 are simple screening tools that identify patients who require more in-depth nutritional assessment and periodic monitoring.

More sophisticated assessments include measurements of body composition, which identify patients with depletion of muscle mass and excess body fat, both of which have been shown to be associated with increased length of stay..$^{13}$ The Subjective Global Assessment is useful in detecting patients with established malnutrition, and the Mini Nutritional Assessment helps to detect patients who need preventive nutritional measures. ${ }^{12}$ Although albumin levels decrease in malnutrition, serum protein levels, including albumin levels, appear to be better correlated with indicators of illness severity and adverse outcomes than with malnutrition. ${ }^{14}$ Thus, the Nutritional Risk Index, which incorporates albumin level and weight loss, is able to capture both poor clinical outcome and nutritional risk. ${ }^{14}$ However, one minor disadvantage is that the index does not allow for "instant" screening, since there is likely to be a lag time before laboratory results are reported.

Nutritional risk continues to be unrecognized and undertreated in clinical practice. Routine screening of all patients is not always done at hospital admission, and nutritional support is often not started for undernourished patients at high nutritional risk. In addition to the impact on morbidity and mortality, disease-related malnutrition also has an economic impact. In one study, the estimated cost of treatment for a patient at nutritional risk was $20 \%$ higher than the average cost of treating the same disease in a patient without nutritional risk. ${ }^{15}$ Given increasing treatment costs and decreasing insurance reimbursements, hospitals need to develop comprehensive strategies to identify and treat malnutrition in patients at hospital admission, to monitor the nutritional status of patients while they are in hospital and to provide nutritional support for those found to have a compromised nutritional status.

Maintaining or improving the nutritional status of patients admitted to hospital will likely improve clinical outcomes and help contain health care costs.
This commentary was solicited and has not been peer reviewed.

Competing interests: None declared.

Contributors: Both authors contributed to the conception and design of the commentary and the writing of the article, and both approved the final version of the paper submitted for publication.

\section{REFERENCES}

1. Stratton RJ, King CL, Stroud MA, et al. "Malnutrition Universal Screening Tool" predicts mortality and length of hospital stay in acutely ill elderly. Br J Nutr 2006; 95:325-30.

2. Wakahara T, Shiraki M, Murase K, et al. Nutritional screening with Subjective Global Assessment predicts hospital stay in patients with digestive diseases. Nutrition 2007; 23:634-9.

3. Sorensen J, Kondrup J, Prokopowicz J, et al. EuroOOPS: an international, multicentre study to implement nutritional risk screening and evaluate clinical outcome. Clin Nutr 2008;27:340-9.

4. Caccialanza R, Klersy C, Cereda E, et al. Nutritional parameters associated with prolonged hospital stay among ambulatory adult patients. CMAJ 2010;182:1843-9.

5. Veterans Affairs Total Parenteral Nutrition Cooperative Study Group. Perioperative total parenteral nutrition in surgical patients. N Engl J Med 1991;325:525-32.

6. Kondrup J, Allison SP, Elia M, et al. ESPEN guidelines for nutrition screening 2002. Clin Nutr 2003;22:415-21.

7. Kyle UG, Kossovsky MP, Karsegard VL, et al. Comparison of tools for nutritional assessment and screening at hospital admission: a population study. Clin Nutr 2006;25:409-17.

8. Detsky AS, McLaughin JR, Baker JP, et al. What is subjective global assessment of nutritional status? JPEN J Parenter Enteral Nutr 1987;11:8-13.

9. Malnutrition Advisory Group (MAG). MAG - guidelines for detection and management of malnutrition. Redditch (UK): British Association for Parenteral and Enteral Nutrition; 2000.

10. Pirlich M, Lochs H. Nutrition in the elderly. Best Pract Res Clin Gastroenterol 2001;15:869-84

11. Guigoz Y. The Mini Nutritional Assessment (MNA) review of the literature: What does it tell us? J Nutr Health Aging 2006;10:466-85.

12. Kyle UG, Genton L, Pichard C. Hospital length of stay (LOS) and nutritional status. Curr Opin Clin Nutr Metab Care 2005;8:397-402.

13. Kyle UG, Pirlich M, Schuetz T, et al. Increased length of hospital stay in underweight and overweight patients at hospital admission: a controlled population study. Clin Nutr 2005;24:133-42.

14. Sullivan DH, Bopp MM, Roberson PK. Protein-energy undernutrition and lifethreatening complications among the hospitalized elderly. J Gen Intern Med 2002; 17:923-32.

15. Amaral TF, Matos LC, Tavares MM, et al. The economic impact of disease-related malnutrition at hospital admission. Clin Nutr 2007;26:778-84.

Correspondence to: Dr. Ursula G. Kyle, Pediatric Critical Care Medicine, Rm. WT6-006, Texas Children's Hospital, 6621 Fannin St., Houston TX 77030, USA; ukyle@bcm.tmc.edu 